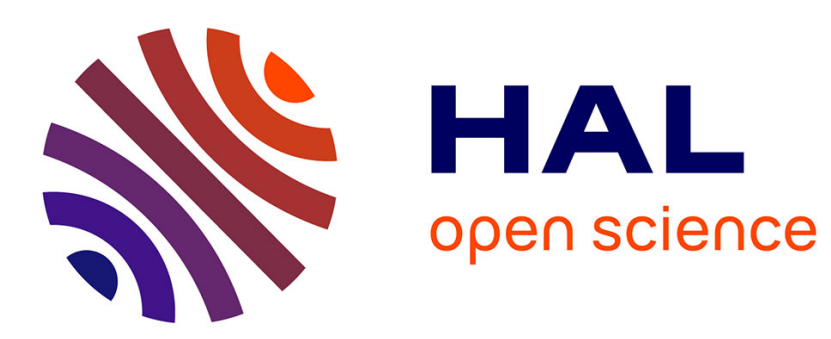

\title{
A numerical method for vibro-acoustic problems with sheared mean flows
}

\author{
Gwenael Gabard, Fabien Treyssède, Mabrouk Ben Tahar
}

\section{To cite this version:}

Gwenael Gabard, Fabien Treyssède, Mabrouk Ben Tahar. A numerical method for vibro-acoustic problems with sheared mean flows. Journal of Sound and Vibration, 2004, 272, pp.991-1011. hal01064463

\section{HAL Id: hal-01064463 \\ https://hal.science/hal-01064463}

Submitted on 16 Sep 2014

HAL is a multi-disciplinary open access archive for the deposit and dissemination of scientific research documents, whether they are published or not. The documents may come from teaching and research institutions in France or abroad, or from public or private research centers.
L'archive ouverte pluridisciplinaire HAL, est destinée au dépôt et à la diffusion de documents scientifiques de niveau recherche, publiés ou non, émanant des établissements d'enseignement et de recherche français ou étrangers, des laboratoires publics ou privés. 


\title{
A NUMERICAL METHOD FOR VIBRO-ACOUSTIC PROBLEMS WITH SHEARED MEAN FLOWS
}

\author{
G. Gabard, F. Treyssède, M. Ben Tahar \\ Laboratoire Roberval UMR 6066, Secteur acoustique \\ Université de Technologie de Compiègne \\ Compiègne, France
}

\begin{abstract}
A model based on Galbrun's equation is proposed to address the problem of vibro-acoustic interactions in sheared flows. The use of a displacement-pressure mixed formulation of Galbrun's equation greatly simplifies the coupling condition formulations and avoid the problem of non-zero frequency spurious modes encountered with displacement based acoustic formulations. This model is applied to duct acoustics. Comparisons with analytical models demonstrate the accuracy of the method. The effects of mean flow shear on acoustic wave propagation in elastic duct are then illustrated.
\end{abstract}

Number of pages: 40 .

Number of tables: 2 .

Number of figures: 13 . 


\section{INTRODUCTION}

Vibro-acoustic interactions are critical aspects in many practical engineering problems and are often found in conjunction with acoustic wave propagation in complex flows (especially in transport engineering).

On the one hand, acoustics of fluid loaded structures in a quiescent fluid have been widely studied for many years. Standard and efficient tools now exist to solve this class of problems [1].

It has been shown that uniform mean flows can significantly change the vibroacoustic behavior of fluid loaded structures $[2,3]$ : an infinite plate may become unstable for certain mean flow speeds (similar conclusions were obtained for finite plates $[4,5]$ and finite cylindrical ducts $[6])$. Such a behavior is often attributed to the negative stiffness added by the acoustic radiation process in the mean flow [7].

On the other hand, the propagation of linear acoustic waves in a non-uniform flow is governed by the linearized Euler equations. Since this set of equations is quite difficult to solve, a simplified model, the full potential theory, has been heavily studied by many investigators [8]. This theory relies on the hypothesis that the acoustic displacements and mean flows are irrotational. But if refraction effects by mean flow shear are to be described, one has to solve the complete linearized Euler equations [9-11].

The influence of sheared mean flows on vibro-acoustic interactions have only been seldom investigated. Pagneux and Aurégan extended Pridmore-Brown's model to infinite ducts with vibrating walls [12]. To address this problem, the present paper introduces an original method based on Galbrun's model [13] for describing acoustic 
waves in a non-uniform mean flow. The coupling conditions are easily formulated since, with a mixed formulation of Galbrun's equation, the acoustic displacement and pressure are explicit variables of the model.

The present paper is organized as follows. Section 2 is intended to introduce the underlying theory of Galbrun's equation. In Section 3, the hypotheses and model for the vibro-acoustic interaction problem are presented and the corresponding variational formulation is derived. The model is then solved in Section 4 with a finite element method. In Section 5, a first group of results is presented to validate the numerical model against an analytical one, then examples illustrate the capabilities of the model.

\section{GALBRUN'S EQUATION}

With the Lagrangian specification, the cinematic of a fluid flow is described by the trajectories $\mathbf{x}(\mathbf{a}, t)$ of the fluid elements. The latter are identified by their positions $\mathbf{a}$ in a reference state so that the independent variables are related to fluid elements. With the Eulerian specification, the velocity field observed for all locations $\mathbf{y}$ in the fluid provides the kinematic of the flow. The independent variables are then associated with a spatial location.

To analyze linear perturbations of fluid dynamic equations, one considers two almost identical flows: a standard one (described by $\mathbf{x}_{0}(t)$ ) and a perturbed one (described by $\mathbf{x}(t)$ ). A quantity (scalar or tensor) measured in the base flow is denoted by $\psi_{0}$ while $\psi$ denotes the same quantity measured in the perturbed flow.

An Eulerian perturbation $\psi^{E}$ is defined as the discrepancy of the measured quan- 
tity between the base and perturbed flows at a given spatial location $\mathbf{y}$. On the other hand, a Lagrangian perturbation $\psi^{L}$ is defined as the discrepancy between the two flows for a given fluid element situated in $\mathbf{x}_{0}$ in the base flow:

$$
\begin{gathered}
\psi^{E}(\mathbf{y}, t)=\psi(\mathbf{y}, t)-\psi_{0}(\mathbf{y}, t) . \\
\psi^{L}\left(\mathbf{x}_{0}, t\right)=\psi(\mathbf{x}, t)-\psi_{0}\left(\mathbf{x}_{0}, t\right) .
\end{gathered}
$$

Since in equation (2), an Eulerian specification of $\psi$ is used to define a perturbation associated with a given fluid element, the quantity $\psi^{L}$ corresponds to a mixed, or Euler-Lagrange, representation.

Assuming small amplitude perturbations, useful properties can be obtained from these definitions. On the one hand, one can derive the following expression relating Eulerian and Lagrangian perturbations valid up to the first order in the perturbation amplitude:

$$
\psi^{E}=\psi^{L}-\mathbf{w} \cdot \nabla \psi_{0}, \quad \text { with } \mathbf{w}=\mathbf{x}(t)-\mathbf{x}_{0}(t),
$$

where $\mathbf{w}$ is the displacement Lagrangian perturbation. Equation (3) shows that Eulerian and Lagrangian perturbations are equivalent when $\psi_{0}$ is uniform in the base flow. On the other hand, it is known that Eulerian perturbation and derivation operations commute (the perturbation of a gradient is the gradient of the perturbation). This does not hold for Lagrangian perturbations:

$$
\begin{gathered}
{\left[\frac{\partial \psi}{\partial t}\right]^{L}=\frac{\partial \psi^{L}}{\partial t}-\frac{\partial \mathbf{w}}{\partial t} \cdot \nabla \psi_{0}} \\
{\left[\frac{\partial \psi}{\partial y_{j}}\right]^{L}=\frac{\partial \psi^{L}}{\partial y_{j}}-\frac{\partial \mathbf{w}}{\partial y_{j}} \cdot \nabla \psi_{0} .}
\end{gathered}
$$

It is worth noting that Eulerian perturbations can be recovered from Lagrangian ones, thought, in general, the opposite is not possible. An in-depth account on 
mixed representation can be found in [14].

In order to obtain Galbrun's wave equation, the definitions and properties of Lagrangian perturbations are applied to fluid dynamic equations for a perfect fluid undergoing an isentropic flow (viscosity and thermal conductivity are neglected). The continuity, momentum and constitutive equations are:

$$
\begin{gathered}
\frac{\partial \rho}{\partial t}+\frac{\partial}{\partial y_{j}}\left(\rho v_{j}\right)=0 \\
\frac{\partial}{\partial t}\left(\rho v_{i}\right)+\frac{\partial}{\partial y_{j}}\left(\rho v_{i} v_{j}\right)+\frac{\partial p}{\partial y_{i}}=0 \\
p=P(\rho, s) .
\end{gathered}
$$

where $\rho$ is the density, $v_{i}$ the velocity components and $p$ the mechanical pressure. Applying the rules (3)-(4) for Lagrangian perturbations to the fluid dynamic equations gives:

$$
\begin{gathered}
\rho^{L}=-\rho_{0} \nabla \cdot \mathbf{w}, \\
\rho^{L} \frac{\mathrm{d}_{0} \mathbf{v}_{0}}{\mathrm{~d} t}+\rho_{0} \frac{\mathrm{d}_{0}^{2} \mathbf{w}}{\mathrm{d} t^{2}}+\nabla p^{L}-\nabla p_{0} \cdot \nabla \mathbf{w}=0 \\
p^{L}=c_{0}^{2} \rho^{L},
\end{gathered}
$$

where $\mathrm{d}_{0} / \mathrm{d} t$ is the material derivative in the mean flow. Combining equations (5) and (7) to eliminate pressure and density variables in equation (6) leads to Galbrun's equation:

$$
\rho_{0} \frac{\mathrm{d}_{0}^{2} \mathbf{w}}{\mathrm{d} t^{2}}-\nabla\left(\rho_{0} c_{0}^{2} \nabla \cdot \mathbf{w}\right)+\nabla p_{0} \nabla \cdot \mathbf{w}-\nabla p_{0} \cdot \nabla \mathbf{w}=0 .
$$

Similar equations were obtained by Hayes [15] and Godin [16]. Galbrun's equation is equivalent to the full linearized Euler equations and has some advantages. 
For isentropic perturbations, the linearized Euler equations require the use of $p$ and $\mathbf{v}$ (see [17]), while Galbrun's equation is expressed for $\mathbf{w}$ only. Moreover, Godin showed that equation (8) derives from a Lagrangian density and derived exact expressions for the energy density and flux $[18,19]$. Such expressions do not exist for the linearized Euler equations.

\section{VIBRO-ACOUSTIC INTERACTION MODEL}

The derivation of the model describing vibro-acoustic interactions is now addressed. The problem at hand is an acoustic domain $\Omega_{a}$ coupled with an elastic

structure $\Omega_{s}$ (see Figure 1). These two domains have a common boundary $\Gamma_{c}$. Two others boundaries are defined: $\Gamma_{a}=\partial \Omega_{a} / \Gamma_{c}$ and $\Gamma_{s}=\partial \Omega_{s} / \Gamma_{c}$.

The structure is considered linear elastic and isotropic with no initial stress and strain. Thus, the structure vibrations are governed by the following set of equations:

$$
\begin{gathered}
\rho_{s} \frac{\partial^{2} \mathbf{u}}{\partial t^{2}}-\nabla \cdot \sigma=\mathbf{f}_{s} \quad \text { on } \Omega_{s}, \\
\mathbf{u}=\overline{\mathbf{u}} \quad \text { or } \quad \sigma \cdot \mathbf{n}=-\mathbf{F}_{s} \quad \text { on } \partial \Omega_{s},
\end{gathered}
$$

where $\rho_{s}$ is the material density, $\mathbf{u}$ the structural displacement, $\sigma$ the stress tensor, $\mathbf{f}_{s}$ the external force density, $\mathbf{F}_{s}$ the boundary forces and $\mathbf{n}$ the inward normal on $\partial \Omega_{s}$.

For the acoustic domain, one considers a perfect fluid undergoing a known, stationary, subsonic flow described by $\left(\rho_{0}, p_{0}, \mathbf{v}_{0}\right)$ given at each point in $\Omega_{a}$. On $\Gamma_{c}$, this mean flow satisfies the slip condition $\mathbf{v}_{0} \cdot \mathbf{n}=0$. The acoustic waves propagating in the mean flow are described by Galbrun's equation (8) which is rewritten: 


$$
\begin{aligned}
& \rho_{0} \frac{\mathrm{d}_{0}^{2} \mathbf{w}}{\mathrm{d} t^{2}}+\nabla p=\mathbf{f}_{a} \quad \text { on } \Omega_{a}, \\
& p+\rho_{0} c_{0}^{2} \nabla \cdot \mathbf{w}=s \quad \text { on } \Omega_{a},
\end{aligned}
$$

where $\mathbf{f}_{a}$ is the external force and $s$ the pressure source. The superscript $L$ on the pressure Lagrangian perturbation $p$ is omitted. For the sake of clarity, the influence of mean flow pressure gradients is neglected here but can be easily taken into account. The use of both pressure and displacement variables will be justified later.

The acoustic waves and structural vibrations are coupled by the geometric and mechanic conditions. Since one considers a perfect fluid, the kinematic compatibility condition states the continuity of the normal displacement on $\Gamma_{c}$ while the mechanic condition implies the normal stress continuity. According to Godin [20], for a structure without initial stress and strain, these conditions reduce to:

$$
\mathbf{w} \cdot \mathbf{n}=\mathbf{u} \cdot \mathbf{n} \quad \text { and } \quad p \mathbf{n}=-\sigma \cdot \mathbf{n} \quad \text { on } \Gamma_{c},
$$

where $\mathbf{n}$ denotes the normal pointing toward the structure.

The vibro-acoustic interaction problem defined by equations (9) to (13) is now expressed as a variational formulation for time harmonic solutions of the form:

$$
(\mathbf{w}, p, \mathbf{u})(\mathbf{x}, t)=(\mathbf{w}, p, \mathbf{u})(\mathbf{x}) \exp (-i \omega t)
$$

The trial functions associated to $\mathbf{w}, p$ and $\mathbf{u}$ are denoted by $\mathbf{w}^{*}, p^{*}$ and $\mathbf{u}^{*}$. After integrating by parts, the following variational formulations are obtained: 


$$
\begin{aligned}
& \int_{\Omega_{a}}-\rho_{0} \frac{\mathrm{d}_{0} \mathbf{w}}{\mathrm{d} t} \cdot \frac{\overline{\mathrm{d}_{0} \mathbf{w}^{*}}}{\mathrm{~d} t}+\mathbf{w} \cdot \overline{\nabla p^{*}}+\nabla p \cdot \overline{\mathbf{w}^{*}}-\frac{p \overline{p^{*}}}{\rho_{0} c_{0}^{2}} \mathrm{~d} \Omega \\
& +\int_{\partial \Omega_{a}} \rho_{0}\left(\mathbf{v}_{0} \cdot \mathbf{n}\right) \frac{\mathrm{d}_{0} \mathbf{w}}{\mathrm{d} t} \cdot \overline{\mathbf{w}^{*}}-\overline{p^{*}} \mathbf{w} \cdot \mathbf{n} \mathrm{d} \Gamma=\int_{\Omega_{a}} \overline{\mathbf{w}^{*}} \cdot \mathbf{f}_{a}-\frac{s \overline{p^{*}}}{\rho_{0} c_{0}^{2}} \mathrm{~d} \Omega \quad \forall\left(\mathbf{w}^{*}, p^{*}\right)(1 \\
& \int_{\Omega_{s}} \sigma: \overline{\varepsilon^{*}}-\omega^{2} \rho_{s} \mathbf{u} \cdot \overline{\mathbf{u}^{*}} \mathrm{~d} \Omega+\int_{\partial \Omega_{s}} \overline{\mathbf{u}^{*}} \cdot \sigma \cdot \mathbf{n} \mathrm{d} \Gamma=\int_{\Omega_{s}} \overline{\mathbf{u}^{*}} \cdot \mathbf{f}_{s} \mathrm{~d} \Omega \quad \forall \mathbf{u}^{*}
\end{aligned}
$$

where the overbar represents the complex conjugate and $\varepsilon$ the symmetric strain tensor. Since one considers time harmonic solutions, the material derivative represents $\mathrm{d}_{0} / \mathrm{d} t=-i \omega+\mathbf{v}_{0} \cdot \nabla$. The boundary integral on $\Gamma_{c}$ in equation (14) is simplified by the slip condition $\mathbf{v}_{0} \cdot \mathbf{n}=0$ for the mean flow. And, with the geometric condition (13a), the normal acoustic displacement is replaced by the normal structural displacement. The mechanic condition (13b) is also used in the boundary integral on $\Gamma_{c}$ in (15) for the structure. Thus, the coupled system variational formulation is obtained:

$$
\begin{aligned}
& \int_{\Omega_{a}}-\rho_{0} \frac{\mathrm{d}_{0} \mathbf{w}}{\mathrm{d} t} \cdot \overline{\frac{\mathrm{d}_{0} \mathbf{w}^{*}}{\mathrm{~d} t}}+\mathbf{w} \cdot \overline{\nabla p^{*}}+\nabla p \cdot \overline{\mathbf{w}^{*}}-\frac{p \overline{p^{*}}}{\rho_{0} c_{0}^{2}} \mathrm{~d} \Omega+\int_{\Omega_{s}} \sigma: \overline{\varepsilon^{*}}-\omega^{2} \rho_{s} \mathbf{u} \cdot \overline{\mathbf{u}^{*}} \mathrm{~d} \Omega \\
& +\int_{\Gamma_{a}} \rho_{0}\left(\mathbf{v}_{0} \cdot \mathbf{n}\right) \frac{\mathrm{d}_{0} \mathbf{w}}{\mathrm{d} t} \cdot \overline{\mathbf{w}^{*}}-\overline{p^{*}} \mathbf{w} \cdot \mathbf{n} \mathrm{d} \Gamma+\int_{\Gamma_{s}} \overline{\mathbf{u}^{*}} \cdot \sigma \cdot \mathbf{n} \mathrm{d} \Gamma-\int_{\Gamma_{c}} \overline{p^{*}} \mathbf{u} \cdot \mathbf{n}+\overline{\mathbf{u}^{*}} \cdot p \mathbf{n} \mathrm{d} \Gamma \\
& =\int_{\Omega_{a}} \overline{\mathbf{w}^{*}} \cdot \mathbf{f}_{a}-\frac{s \overline{p^{*}}}{\rho_{0} c_{0}^{2}} \mathrm{~d} \Omega+\int_{\Omega_{s}} \overline{\mathbf{u}^{*}} \cdot \mathbf{f}_{s} \mathrm{~d} \Gamma \quad \forall\left(\mathbf{w}^{*}, p^{*}, \mathbf{u}^{*}\right),
\end{aligned}
$$

The use of a mixed pressure-displacement formulation for the acoustic waves is justified by two arguments.

First, the use of acoustic displacement and pressure variables leads to a simple formulation of the coupling conditions. With displacement based formulations, special treatments are required at the fluid-structure interface $\Gamma_{c}$ in order to take the mechanical coupling condition into account $[21,22]$. This is not the case with 
the mixed formulation (16) since the variables $\mathbf{w}, p$ and $\mathbf{u}$ involved in equation (13) are explicit variables of the model.

Secondly, it is worth noting that with no mean flow Galbrun's equation (8) reduces to the standard displacement based acoustic equation. A well-known issue concerning displacement based acoustic formulation is the occurrence of non-zero frequency rotational spurious modes which are purely numerical solutions without physical meaning [23]. Hamdi et al. proposed a penalty method to overcome this difficulty [24], but the spurious modes are shifted to higher frequencies and not removed [25]. Bermúdez et al. used edge elements to ensure that the displacement field satisfies the irrotationality condition [22]. Wang and Bathe showed that mixed pressure-displacement finite elements satisfying the so-called inf-sup condition are not polluted by spurious modes [26]. To satisfy this sufficient condition for stability a mixed formulation has to be solved with carefully chosen finite element interpolations (for more informations on this kind of elements see [27] and [28]).

Spurious numerical modes have also been encountered with Galbrun's equation (8). Bonnet et al. [29] proposed a regularisation technique for uniform mean flows. Peyret also proposed the use of edge elements to describe the displacement field [30]. A penalty method is not applicable here since with sheared mean flows the acoustic displacement may be rotational.

In the present paper, Wang and Bathe's approach is followed by employing a mixed formulation of Galbrun's equation with mean flow together with finite elements satisfying the inf-sup condition. The originality of Galbrun's equation is that the pressure-displacement relationship given by (12) is not altered by the pres- 
ence of flow and is strictly identical to the no-flow case. Furthermore, the infsup condition properties are closely related to the accurate approximation of this pressure-displacement relation. This enables to directly apply the inf-sup condition to a Galbrun-based formulation. Thus, under the assumption that the additional operators introduced by the presence of flow (terms with $\mathbf{v}_{0} \cdot \nabla$ ) does not alter convergence properties of elements satisfying the inf-sup condition, it is expected that the proposed mixed method for solving Galbrun's equation is robust. Without structural coupling, these expectations have been confirmed by comprehensive numerical tests detailed in reference [31].

\section{FINITE ELEMENT MODEL}

In this section, a finite element model is used to solve the variational formulation (16) for axisymmetric ducts. The solutions are written:

$$
(\mathbf{w}, p, \mathbf{u})(r, \theta, z, t)=(\mathbf{w}, p, \mathbf{u})(r, z) \exp (i m \theta-i \omega t)
$$

where $m$ is the azimuthal mode number.

In the $(r, z)$ plane, the computational acoustic domain is approximated with linear triangular elements. The pressure interpolation is linear while the displacement interpolation uses linear functions plus a 'bubble' function (this element is known as $P_{1}^{+}-P_{1}$ or $4-3 c$ ). With no mean flow, Wang and Bathe have demonstrated the stability of this element which satisfies the inf-sup condition $[27, p .300]$.

The structure $\Omega_{s}$ corresponds to the duct walls which are thin shell described with Mindlin's theory. The duct walls are approximated by linear elements. On each element, displacements and rotations are described with linear functions. A 
complete description of this shell element is given in [32,p.100].

After assembling the element contributions, the following linear system is obtained:

$$
\left[\begin{array}{ccc}
\left(\mathbf{M}_{a}+\mathbf{B}_{a}\right) & \mathbf{D}_{a} & \mathbf{0} \\
\left(\mathbf{D}_{a}^{\mathrm{T}}+\mathbf{E}_{a}\right) & \mathbf{K}_{a} & \mathbf{C} \\
\mathbf{0} & \mathbf{C}^{\mathrm{T}} & \left(\mathbf{K}_{s}+\mathbf{M}_{s}\right)
\end{array}\right]\left[\begin{array}{c}
\widehat{\mathbf{w}} \\
\widehat{\mathbf{p}} \\
\widehat{\mathbf{u}}
\end{array}\right]=\left[\begin{array}{c}
\mathbf{F}_{w} \\
\mathbf{F}_{p} \\
\mathbf{F}_{u}
\end{array}\right]
$$

where $\widehat{\mathbf{w}}, \widehat{\mathbf{p}}$ and $\widehat{\mathbf{u}}$ denote the acoustic displacement, pressure and structural displacement unknown vectors respectively. The matrices introduced in equation (18) are defined by:

$$
\begin{aligned}
& \widehat{\mathbf{w}}^{* \mathrm{~T}} \mathbf{M}_{a} \widehat{\mathbf{w}}=\int_{\Omega_{a}}-\rho_{0} \frac{\mathrm{d}_{0} \mathbf{w}}{\mathrm{d} t} \cdot \frac{\overline{\mathrm{d}_{0} \mathbf{w}^{*}}}{\mathrm{~d} t} \mathrm{~d} \Omega, \quad \widehat{\mathbf{p}}^{* \mathrm{~T}} \mathbf{K}_{a} \widehat{\mathbf{p}}=-\int_{\Omega_{a}} \frac{\overline{p^{*} p}}{\rho_{0} c_{0}^{2}} \mathrm{~d} \Omega, \\
& \widehat{\mathbf{w}}^{* \mathrm{~T}} \mathbf{D}_{a} \widehat{\mathbf{p}}=\int_{\Omega_{a}} \overline{\mathbf{w}^{*}} \cdot \nabla p \mathrm{~d} \Omega, \quad \quad \widehat{\mathbf{w}}^{* \mathrm{~T}} \mathbf{B}_{a} \widehat{\mathbf{w}}=\int_{\Omega_{a}} \rho_{0}\left(\mathbf{v}_{0} \cdot \mathbf{n}\right) \frac{\mathrm{d}_{0} \mathbf{w}}{\mathrm{d} t} \cdot \overline{\mathbf{w}^{*}} \mathrm{~d} \Omega, \\
& \widehat{\mathbf{p}}^{* \mathrm{~T}} \mathbf{E}_{a} \widehat{\mathbf{w}}=-\int_{\Gamma_{a}} \overline{p^{*}} \mathbf{w} \cdot \mathbf{n} \mathrm{d} \Gamma, \quad \widehat{\mathbf{p}}^{* \mathrm{~T}} \mathbf{C} \widehat{\mathbf{u}}=-\int_{\Gamma_{c}} \overline{p^{*}} \mathbf{u} \cdot \mathbf{n} \mathrm{d} \Gamma, \\
& \widehat{\mathbf{u}}^{* \mathrm{~T}} \mathbf{K}_{s} \widehat{\mathbf{u}}=\int_{\Omega_{s}} \sigma: \overline{\varepsilon^{*}} \mathrm{~d} \Omega, \quad \widehat{\mathbf{u}}^{* \mathrm{~T}} \mathbf{M}_{s} \widehat{\mathbf{u}}=-\omega^{2} \int_{\Omega_{s}} \rho_{s} \overline{\mathbf{u}^{*}} \cdot \mathbf{u} \mathrm{d} \Omega .
\end{aligned}
$$

It is worth noting that $\mathbf{M}_{a}, \mathbf{K}_{a}, \mathbf{K}_{s}$ and $\mathbf{M}_{s}$ are hermitian matrices. The sparse system (18) can be readily solved to obtain the finite element solution.

\section{VALIDATION}

To demonstrate the validity and accuracy of the numerical model, it is tested against two analytical models which are complementary. The first one considers finite length elastic duct with uniform flow and the external solicitation is applied on the structure. On the other hand, the second model [12] is an extension of Pridmore-Brown's equation and handles infinite elastic duct with sheared flows. 


\subsection{FINITE DUCT WITH UNIFORM MEAN FLOW}

One considers an annular duct carrying an uniform axial mean flow (see Figure 2 ). The duct outer wall is an elastic thin shell while the inner wall is rigid. At both ends of the duct, all rotations and displacement are prescribed to zero except axial displacements and azimuthal rotations. A radial, uniform, harmonic force $\left(1 \mathrm{~N} / \mathrm{m}^{2}\right)$ is applied on the shell and the acoustic pressure is set to zero at the inlet $(z=0)$ and outlet $(z=L)$. Thought it does not represent a practical situation, this problem is useful since it is possible to derive an analytical solution. The details of this analytical model are given in Appendix A and Table 1 summarizes the duct and fluid properties.

Axisymmetric solutions $(m=0)$ are sought in the two following cases: (i) with no flow at $245 \mathrm{~Hz}$ and (ii) with a $0.3 \mathrm{Mach}$ number flow at $318 \mathrm{~Hz}$. These two cases exhibit a strong coupling between an acoustic cavity mode and a structural mode.

The finite element mesh used for the computations is shown on Figure 2. The mesh is refined near the shell in order to better describe its vibrations. The shell is discretized with 80 elements and the acoustic domain with 1000 elements. This corresponds approximately to 13 elements per wavelength in the two cases.

Comparisons of the analytical and numerical results are shown on Figures 3 to 5. A good accuracy is achieved in the two cases both for acoustic pressures and structural displacement amplitudes. The small deviation of the numerical result from the analytical solution on Figure 5 can be attributed to the very small dissipation added by the numerical schemes. The effect of the numerical dissipation is noticeable here since there is a strong coupling between the acoustical and structural modes. 


\subsection{INFINITE DUCT WITH SHEARED MEAN FLOW}

The numerical method is also validated with the model proposed by Pagneux and Aurégan [12]. It represents an extension of Pridmore-Brown's equation [33] by providing a boundary condition accounting for the coupling with the elastic duct wall. It is limited to infinite ducts but it takes the duct wall elasticity and the mean flow shear into account. Details on this analytical model are provided in Appendix B.

The computational domain and the finite element mesh are similar to that described on Figure 2 and the problem parameters are also given by Table 1. Here, the same approach as in reference [31] is used to validate the numerical model. At the inlet $(z=0)$, the displacement is prescribed in order to simulate an incoming acoustic mode provided by the analytical model. The propagation impedance of the acoustic mode is applied at the outlet $(z=L)$ to implement a non-reflecting boundary condition. This method can be inaccurate if other acoustic modes or hydrodynamic disturbances are present. However, the examples presented below are believed not to be polluted by important spurious reflections at the outlet. A more general technique such as a modal decomposition of the solution at the outlet [34] should overcome this limitation. At both ends of the duct, the structural displacements and rotations given by the analytical model are imposed.

Results obtained with the analytical model and the finite element method are presented for the normal mode defined by $m=0, n=1$ at $800 \mathrm{~Hz}$ with uniform and sheared mean flows. For the latter case, downstream and upstream propagations are investigated with a parabolic boundary layer profile (with a $10 \%$ thickness). The 
averaged Mach number in a section is 0.3 . In all cases, one finds good agreement between the two models both for the acoustic pressure (Figure 6) and the structural displacement (Figure 7). Thus, the acoustic mode propagation in the sheared flow and the vibro-acoustic coupling with the duct wall are accurately described by the finite element model.

Furthermore, structural displacements (Figure 7) provide interesting insight on the effect of acoustic refraction by mean flow shear on vibro-acoustic coupling. It is well-known that flow shear modifies downstream acoustic propagation by deviating the acoustic energy towards duct walls. For upstream propagation in shear flow, the acoustic energy is concentrated near the duct axis [35]. This effect explains the fact that the vibration amplitude is much larger in the downstream propagation case. Due to the acoustic energy deviation near the duct, the coupling with the elastic duct wall is more important.

\section{EXAMPLES}

In this section, the capabilities of the numerical method to solve realistic problems with higher order modes $(m \neq 0)$ and complex geometries is illustrated. The following examples also represents situations where it is necessary to describe accurately both vibro-acoustic interactions and wave propagation in sheared flows.

A cylindrical elastic duct which carries an axial flow is placed between two infinite rigid ducts (see Figure 8). The computational domain comprises the 1 meter long elastic duct and two portions of the rigid ducts (half a meter each). The duct boundary conditions are the same as for the validation problem. At the inlet, an 
incoming acoustic mode is simulated by prescribing the corresponding displacement field, the pressure is not imposed. The acoustic mode displacement profiles at the inlet section are obtained with Pridmore-Brown's model for a rigid duct [33]. At the outlet, the non-reflecting condition based on the modal impedance is used.

In the following results, the incoming acoustic mode defined by $m=4, n=1$ at $570 \mathrm{~Hz}$ is considered. Other parameters of the problem are given in Table 2. Figure 8 shows the finite element mesh. The shell is discretized with 60 elements and the acoustic domain with 2300 elements.

To demonstrate the influence of the elastic duct on the acoustic mode propagation with no flow, Figure 9 compares pressure amplitudes obtained with rigid and elastic ducts. The acoustic mode, otherwise propagating, is reflected when interacting with the elastic duct.

Figure 10 shows the solutions obtained with uniform and parabolic flows at a 0.2 averaged Mach number. Compared with the no flow case, the uniform mean flow clearly modifies the pressure distribution in the duct by changing the acoustic axial wave number and so weakening the coupling of acoustic and structural modes. The results obtained with the parabolic flow are slightly different from the uniform flow case.

The solutions obtained with uniform and parabolic flows with a -0.2 averaged Mach number (the fluid flows downward) are given on Figure 11. Compared to the downstream propagation case, the upstream propagation is less influenced by the uniform mean flow. On the contrary, the upstream propagation is much more influenced by the flow shear. It can be noted that results obtained for the upstream 
propagation in the parabolic flow are similar to the no flow case.

The last example is presented to demonstrate that the proposed model can handle arbitrary geometry. The duct and acoustic mode are the same as above except that the elastic duct radius is given by (see figure 12):

$$
R(z)=0.9-0.1 \cos [\pi(z-0.5)] \quad \text { for } 0.5<z<1.5 \text {. }
$$

Figure 13 shows pressure modulus at $1015 \mathrm{~Hz}$ with no flow and with an upward incompressible irrotational flow (with a 0.2 Mach number at the inlet). Thought this irrotational mean flow does not generate refraction effect (by boundary layer for instance) it is found to have a noticeable impact on the acoustic propagation.

\section{SUMMARY}

To model vibro-acoustic interactions with shear mean flow, a method based on Galbrun's equation is proposed. To avoid spurious numerical modes, Galbrun's equation is rewritten as a mixed displacement-pressure problem and a finite element discretization satisfying the inf-sup condition is chosen. The mixed formulation also afford a simple way to take the fluid-structure coupling conditions into account. Compared to similar models based on the full potential theory, the present approach has the ability to describe refraction effects by the mean flow shear. It has been shown that the small modifications of a ducted acoustic mode introduced by the flow shear can significantly change the behavior of the coupled fluid-elastic duct system.

Though the method has been applied to duct acoustics only, it is applicable to a wide range of problems. 


\section{REFERENCES}

1. H.J.-P. MORAnd and R. OHAYon 1992 Interactions fluides-structures. Masson.

2. P.R. Brazier-Smith and J.F. Scott 1984 Wave Motion 6, 547-560. Stability of fluid flow in the presence of a compliant surface.

3. D.C. Crighton and J.E. Oswell 1991 Phil. Trans. R. Soc. London 335, 557-592. Fluid loading with mean flow. I. Response of an elastic plate to localized excitation.

4. F. Sgard, N. Atalla and J. Nicolas 1994 AIAA Journal 32, 2351-2358. Coupled BEM-FEM approach for mean flow effects on vibro-acoustic behavior of planar structures.

5. S.F. Wu and J. ZHu 1999 Journal of the Acoustical Society of America 105, 732-742. Bifurcation and chaos in flexural vibration of a baffled plate in mean flow.

6. N. OuelaA, B. Laulagnet and J.L. Guyader 1994 Acta Acustica 2, 275-289. Étude vibro-acoustique d'une coque cylindrique finie remplie de fluide en mouvement uniforme.

7. S.F. Wu and L. Maestrello 1995 AIAA Journal 33, 13-19. Responses of finite baffled plate to turbulent flow excitation.

8. R.K. Sigman, R.K. MajJigi and B.T. Zinn 1978 AIAA Journal 16, 1139-1145. Determination of turbofan inlet acoustics using finite elements.

9. R.J. Astley and W. Eversman 1979 Journal of Sound and Vibration 65, 61-74. A finite element formulation of the eigenvalue problem in lined ducts with flow.

10. C. Bailly and D. JuvÉ 2000 AIAA Journal 38, 22-29. Numerical solution of acoustic propagation problems using linearized Euler equations.

11. E. Longatte and P. Lafon 2000 AIAA Journal 38, 389-394. Computation of acoustic propagation in two-dimensional sheared ducted flows.

12. V. Pagneux and Y. Aurégan. Acoustic modes in duct with parallel shear flow and vibrating walls. In 4th AIAA/CEAS Aeroacoustics Conference pages 441-447 1998. AIAA paper $98-2281$.

13. H. Galbrun 1931 Propagation d'une onde sonore dans l'atmosphère terrestre et théorie 
des zones de silence. Gauthier-Villars.

14. B. PoiréE. Les équations de l'acoustique linéaire et non-linéaire dans les fluides en mouvement. Thèse d'état Université Pierre et Marie Curie, Paris 61982.

15. W. HaYes 1970 Proc. Roy. Soc. London A, 187-208. Conservation of action and modal waves action.

16. O.A. Godin 1996 Physics-Doklady 41, 521-524. On the small-amplitude waves in an inhomogeneous moving medium.

17. D. Blokhintzev 1946 Journal of the Acoustical Society of America 18, 322-328. The propagation of sound in an inhomogeneous and moving medium I.

18. O.A. Godin 1997 Wave Motion 25, 143-167. Reciprocity and energy theorems for waves in a compressible inhomogeneous moving fluid.

19. C. Peyret and G. ÉLias 2001 Journal of the Acoustical Society of America 110, 661-668. Finite-element method to study harmonic aeroacoustics problems.

20. O.A. Godin 1997 Acoustical Physics 43, 688-693. Reciprocity relations and energy conservation for waves in the system: inhomogeneous fluid flow-anisotropic solid body.

21. M. Ben Tahar and E. Goy. Resolution of vibroacoustic problem in the presence of a non-uniform mean flow. In 4th AIAA/CEAS Aeroacoustics Conference 1998. Paper 98-2215.

22. A. Bermúdez, L. Hervella-Nieto and R. Rodríguez 1999 Journal of Sound and Vibration 219, 279-306. Finite element computation of three-dimensional elastoacoustic vibrations.

23. L. Kiefling and G.C. Fleng 1976 AIAA Journal 14, 199-203. Fluid-structure finite element vibrational analysis.

24. M.A. Hamdi, Y. Ousset and G. Verchery 1978 Int. J. Numer. Methods Eng. 13, 139-150. A displacement method for the analysis of vibrations of coupled fluid-structure systems.

25. L.G. Olson and K.-J. Bathe 1983 Nucl. Eng. Des. 76, 137-151. A study of displacement-based fluid finite elements for calculating frequencies of fluid and fluid- 
structure system.

26. X. WAng and K.-J. BAthe 1997 International Journal for Numerical Methods in Engineering 40, 2001-2017. Displacement/pressure based mixed finite element formulations for acoustic fluid-structure interaction problems.

27. K.-J. BAthe 1996 Finite element procedures. Academic Press.

28. F. Brezzi and M. Fortin 1991 Mixed and hybrid finite element methods. SpringerVerlag.

29. A.-S. Bonnet-Ben Dhia, G. Legendre and E. Lunéville 2001 C. R. Acad. Sci. Paris IIb, 601-606. Analyse mathématique de l'équation de Galbrun en écoulement uniforme.

30. C. Peyret. Mise en oeuvre d'éléments finis d'arêtes pour résoudre l'équation de Galbrun. In Actes des 2 Journées sur l'acoustique de Galbrun Arcachon, France April 2001.

31. F. Treyssède, G. Gabard and M. Ben Tahar 2003 Journal of the Acoustical Society of America 113, 705-716. A mixed finite element method for acoustic propagation in moving fluids based on an Eulerian-Lagrangian description.

32. J.-L. Batoz and G. Dhatt 1992 Modélisation des structures par éléments finis Coques. Hermès, Paris.

33. D.C. Pridmore-Brown 1958 Journal of Fluid Mechanics 4, 393-406. Sound propagation in a fluid flowing through an attenuating duct.

34. W. Eversman 1991 NASA Reference Publication 1258. Aeroacoustics of flight vehicules: theory and practice, Vol. 2: Noise control Theoretical models for duct acoustic propagation and radiation.

35. P. Mungur and H.E. Plumbee 1969 NASA SP-207. Propagation and attenuation of sound in a soft-walled annular duct containing a sheared flow. 


\section{APPENDIX A: ANALYTICAL MODEL FOR A FINITE DUCT}

An analytical model can be derived for axisymmetric solutions $m=0$ of the validation problem in Section 5.1. The duct outer wall is a thin shell: $h / R_{2} \ll 1$. At both ends of the duct all rotations and displacement are prescribed to zero except axial displacements and azimuthal rotations. Assuming that the duct axial displacement is small compared to the radial displacement, it can be shown that the duct radial displacement $u$ is governed by the following equations:

$$
\begin{gathered}
D \frac{\partial^{4} u}{\partial z^{4}}+\frac{E h}{R_{2}^{2}} u+\rho_{s} h \frac{\partial^{2} u}{\partial t^{2}}=f_{e}+p \quad \text { with } D=\frac{E h^{3}}{12\left(1-\nu^{2}\right)}, \\
u=0, \quad \frac{\partial^{2} u}{\partial z^{2}}=0, \quad \text { for } z=0, L .
\end{gathered}
$$

The acoustic pressure in the duct is governed by the convected wave equation:

$$
\begin{gathered}
\frac{1}{c_{0}^{2}} \frac{\partial^{2} p}{\partial t^{2}}+2 \frac{M}{c_{0}} \frac{\partial^{2} p}{\partial z \partial t}+M^{2} \frac{\partial^{2} p}{\partial z^{2}}-\Delta p=0 \\
p=0, \quad \text { for } z=0, L
\end{gathered}
$$

where $M$ is the Mach number of the axial, uniform mean flow in the duct. The geometric coupling condition is expressed by:

$$
\begin{gathered}
\frac{\partial p}{\partial r}=0 \quad \text { for } r=R_{1}, \\
\frac{\partial p}{\partial r}=-\rho_{0} \frac{\mathrm{d}_{0}^{2} u}{\mathrm{~d} t^{2}} \quad \text { for } r=R_{2}
\end{gathered}
$$

For an harmonic force $f_{e}$, acoustic pressure and radial structural displacement can be written:

$$
p(r, z, t)=\sum_{a=0}^{\infty} \sum_{b=1}^{\infty} \alpha_{a b} p_{a b}(r, z) e^{-i \omega t}, \quad u(z, t)=\sum_{c=1}^{\infty} \beta_{c} u_{c}(z) e^{-i \omega t}
$$


where $a$ and $b$ are the radial and axial mode number, respectively. The modes are given by:

$$
\begin{gathered}
p_{a b}(r, z)=A_{a} S_{0}\left(k_{r a} r\right) \sin \left(\frac{b \pi z}{L}\right) \exp \left(\frac{-i k M z}{1-M^{2}}\right) \quad a>0, b>0, \\
p_{0 b}(r, z)=A_{0} \sin \left(\frac{b \pi z}{L}\right) \exp \left(\frac{-i k M z}{1-M^{2}}\right) \quad b>0, \\
u_{c}(z)=\sqrt{\frac{1}{\pi R_{2} L} \sin \left(\frac{c \pi z}{L}\right) \quad c>0,} \\
A_{0}=\sqrt{\frac{2 \sqrt{1-M^{2}}}{\pi L\left(R_{2}^{2}-R_{1}^{2}\right)}}, \quad A_{a}=\sqrt{\frac{2 \sqrt{1-M^{2}}}{\pi L\left[R_{2}^{2} S_{0}^{2}\left(k_{r a} R_{2}\right)-R_{1}^{2} S_{0}^{2}\left(k_{r a} R_{1}\right)\right]}},
\end{gathered}
$$

with $k=\omega / c_{0}$ and

$$
S_{0}\left(k_{r a} r\right)=\mathrm{J}_{0}\left(k_{r a} r\right)-\frac{\mathrm{J}_{0}^{\prime}\left(k_{r a} R_{1}\right)}{\mathrm{Y}_{0}^{\prime}\left(k_{r a} R_{1}\right)} \mathrm{Y}_{0}\left(k_{r a} r\right)
$$

$\mathrm{J}_{0}$ and $\mathrm{Y}_{0}$ are the zeroth order Bessel functions of the first and second kind, respectively. The radial wave numbers $k_{r a}$ are the solutions of the caracteristic equation:

$$
\mathrm{J}_{0}^{\prime}\left(k_{r} R_{1}\right) \mathrm{Y}_{0}^{\prime}\left(k_{r} R_{2}\right)=\mathrm{J}_{0}^{\prime}\left(k_{r} R_{2}\right) \mathrm{Y}_{0}^{\prime}\left(k_{r} R_{1}\right) .
$$

The generalized variables $\alpha_{a b}$ and $\beta_{c}$ are defined by a set of linear equations:

$$
\begin{aligned}
& \Omega_{a b}^{A} \alpha_{a b}+\sum_{c} C_{a b c}^{A} \beta_{c}=0 \quad \forall a, b, \\
& \Omega_{c}^{S} \beta_{c}+\sum_{a, b} C_{a b c}^{S} \alpha_{a b}=f_{c} \quad \forall c .
\end{aligned}
$$

The coefficients of this linear system are given by:

$$
\Omega_{a b}^{A}=k_{r a}^{2}+\frac{b^{2} \pi^{2}}{L^{2}}\left(1-M^{2}\right)-\frac{k^{2}}{\left(1-M^{2}\right)}, \quad \Omega_{c}^{S}=D\left(\frac{c \pi}{L}\right)^{4}+\frac{E h}{R_{2}^{2}}-\rho_{s} \omega^{2} .
$$

For $a>0$, one has the following expressions:

$$
\begin{aligned}
& C_{a b c}^{A}=\frac{2 \pi \rho_{0} R_{2} A_{a} S_{0}\left(k_{r a} R_{2}\right)}{\sqrt{1-M^{2}} \sqrt{\pi R_{2} L}}\left\{2 i \omega c_{0} M \frac{c \pi}{L} L_{b c}+\left[\omega^{2}+c_{0}^{2} M^{2}\left(\frac{c \pi}{L}\right)^{2}\right] K_{b c}\right\}, \\
& C_{a b c}^{S}=\frac{2 \pi R_{2}}{\sqrt{\pi R_{2} L}} A_{a} S_{0}\left(k_{r a} R_{2}\right) \bar{K}_{b c},
\end{aligned}
$$


where

$$
\begin{gathered}
K_{b c}=\frac{2 i \pi^{2} B b c}{L} \frac{e^{i\left(A_{+}-B\right)}-1}{\left(A_{+}^{2}-B^{2}\right)\left(A_{-}^{2}-B^{2}\right)}, \\
L_{b c}=\frac{\pi B}{L}\left(1-e^{i\left(A_{+}-B\right)}\right) \frac{A_{+} A_{-}-B^{2}}{\left(A_{+}^{2}-B^{2}\right)\left(A_{-}^{2}-B^{2}\right)}, \\
A_{ \pm}=(b \pm c) \pi, \quad B=\frac{k M L}{1-M^{2}} .
\end{gathered}
$$

For the case $a=0$, the expressions above are replaced by:

$$
\begin{aligned}
C_{0 b c}^{A} & =\frac{2 \pi \rho_{0} R_{2} A_{0}}{\sqrt{1-M^{2}} \sqrt{\pi R_{2} L}}\left\{2 i \omega c_{0} M \frac{c \pi}{L} L_{b c}+\left[\omega^{2}+c_{0}^{2} M^{2}\left(\frac{c \pi}{L}\right)^{2}\right] K_{b c}\right\}, \\
C_{0 b c}^{S} & =\frac{2 \pi R_{2}}{\sqrt{\pi R_{2} L}} A_{0} \bar{K}_{b c} .
\end{aligned}
$$

With no mean flow, $K_{b c}$ is given by:

$$
K_{b c}=\frac{L}{2} \delta_{b c}
$$

The right-hand side of equations (A.2) is given by:

$$
f_{c}=2 \pi R_{2} \int_{0}^{L} u_{c}(z) f_{e}(z) \mathrm{d} z .
$$

The infinite sums in equation (A.1) are truncated and the finite dimensional linear system (A.2) is solved for the generalized variables $\alpha_{a b}$ and $\beta_{c}$. Acoustic pressures and structural displacements are then recovered by means of the modal decompositions (A.1).

\section{APPENDIX B: EXTENSION OF PRIDMORE-BROWN'S EQUATION}

Pagneux and Aurégan have extended Pridmore-Brown's equation [33] by deriving a boundary condition which accounts for the duct wall elasticity [12]. When seeking for normal modes defined by

$$
\left(u_{r}, u_{z}, p\right)(r, z, t)=\left(u_{r}, u_{z}, p\right)(r) \exp \left(i k_{z} z-i \omega t\right)
$$


Pridmore-Brown's equation in an axisymmetric duct with axial mean flow $\mathbf{v}_{0}=$ $v_{0}(r) \mathbf{e}_{z}$ can be written:

$$
\frac{\partial^{2} p}{\partial r^{2}}+\left(\frac{1}{r}+\frac{2 k_{z}}{\omega-k_{z} v_{0}} \frac{\partial v_{0}}{\partial r}\right) \frac{\partial p}{\partial r}+\left[\left(\frac{\omega-k_{z} v_{0}}{\omega}\right)^{2}-k_{z}^{2}\right] p=0
$$

It is worth noting that this equation can be obtained from Galbrun's equation (8). Equation (B.1) is generally supplemented by rigid- or lined-wall boundary conditions. The boundary condition proposed in [12] is obtained directly from the equations for an axisymmetric thin shell based on Kirchhoff theory:

$$
\begin{gathered}
\left(\frac{\omega^{2}}{c_{s}^{2}}-k_{z}^{2}\right) u_{z}+i k_{z} \frac{\nu}{R} u_{r}=0, \\
i k_{z} \frac{\nu}{R} \frac{E h}{1-\nu^{2}} u_{z}+\left(\frac{E h}{1-\nu^{2}} \frac{1}{R^{2}}+k_{z}^{4} D-\rho_{s} h \omega^{2}\right) u_{r}=p,
\end{gathered}
$$

with $c_{s}^{2}=E / \rho_{s} /\left(1-\nu^{2}\right)$. Furthermore, the pressure gradient can be related to the structure displacement by means of Euler equations and the normal displacement continuity:

$$
\frac{\partial p}{\partial r}=\rho_{0}\left(\omega-k_{z} v_{0}\right)^{2} u_{r}
$$

Upon eliminating $u_{r}$ and $u_{z}$ in equations (B.2), (B.3) and (B.4), one can obtain the following boundary condition for the pressure in the duct:

$$
\left[\rho_{s} h \frac{\nu^{2} k_{z}^{2} c_{s}^{2}}{R^{2}}+\left(\frac{\omega^{2}}{c_{s}^{2}}-k_{z}^{2}\right)\left(\rho_{s} \frac{h}{R^{2}} c_{s}^{2}-\rho_{s} h \omega^{2}+D k_{z}^{4}\right)\right] \frac{\partial p}{\partial r}=\rho_{0}\left(\omega-k_{z} v_{0}\right)^{2}\left(\frac{\omega^{2}}{c_{s}^{2}}-k_{z}^{2}\right) p .
$$

By solving Pridmore-Brown's equation with this boundary condition at $r=R$, one can determine the normal modes of an infinite elastic duct carrying an axial sheared mean flow. In this paper, the normal modes are normalized by the integral over a section of the squared pressure. 
TABLE 1

Parameters of the validation test

\begin{tabular}{|c|c|c|c|}
\hline & meter & Symbol & Value \\
\hline \multirow[t]{8}{*}{ Duct } & Inner radius & $R_{1}$ & $0.2 \mathrm{~m}$ \\
\hline & Outer radius & $R_{2}$ & $1 \mathrm{~m}$ \\
\hline & Length & $L$ & $2 \mathrm{~m}$ \\
\hline & Thickness & $h$ & $1 \mathrm{~mm}$ \\
\hline & Material & & aluminum \\
\hline & Density & $\rho_{s}$ & $2700 \mathrm{~kg} / \mathrm{m}^{3}$ \\
\hline & Young's modulus & $E$ & $7.1 \times 10^{10} \mathrm{~N} / \mathrm{m}^{2}$ \\
\hline & Poisson's ratio & $\nu$ & 0.3 \\
\hline \multirow[t]{2}{*}{ Fluid } & Density & $\rho_{0}$ & $1.2 \mathrm{~kg} / \mathrm{m}^{3}$ \\
\hline & Sound speed & $c_{0}$ & $340 \mathrm{~m} / \mathrm{s}$ \\
\hline
\end{tabular}


TABLE 2

Parameters of the example problem

\begin{tabular}{|c|c|c|c|}
\hline & eter & Symbol & Value \\
\hline \multirow[t]{7}{*}{ Elastic duct } & Radius & $R$ & $1 \mathrm{~m}$ \\
\hline & Length & $L$ & $1 \mathrm{~m}$ \\
\hline & Thickness & $h$ & $2 \mathrm{~mm}$ \\
\hline & Material & & aluminum \\
\hline & Density & $\rho_{s}$ & $2700 \mathrm{~kg} / \mathrm{m}^{3}$ \\
\hline & Young's modulus & $E$ & $7.1 \times 10^{10} \mathrm{~N} / \mathrm{m}^{2}$ \\
\hline & Poisson's ratio & $\nu$ & 0.3 \\
\hline \multirow[t]{2}{*}{ Fluid } & Density & $\rho_{0}$ & $1.2 \mathrm{~kg} / \mathrm{m}^{3}$ \\
\hline & Sound speed & $c_{0}$ & $340 \mathrm{~m} / \mathrm{s}$ \\
\hline
\end{tabular}




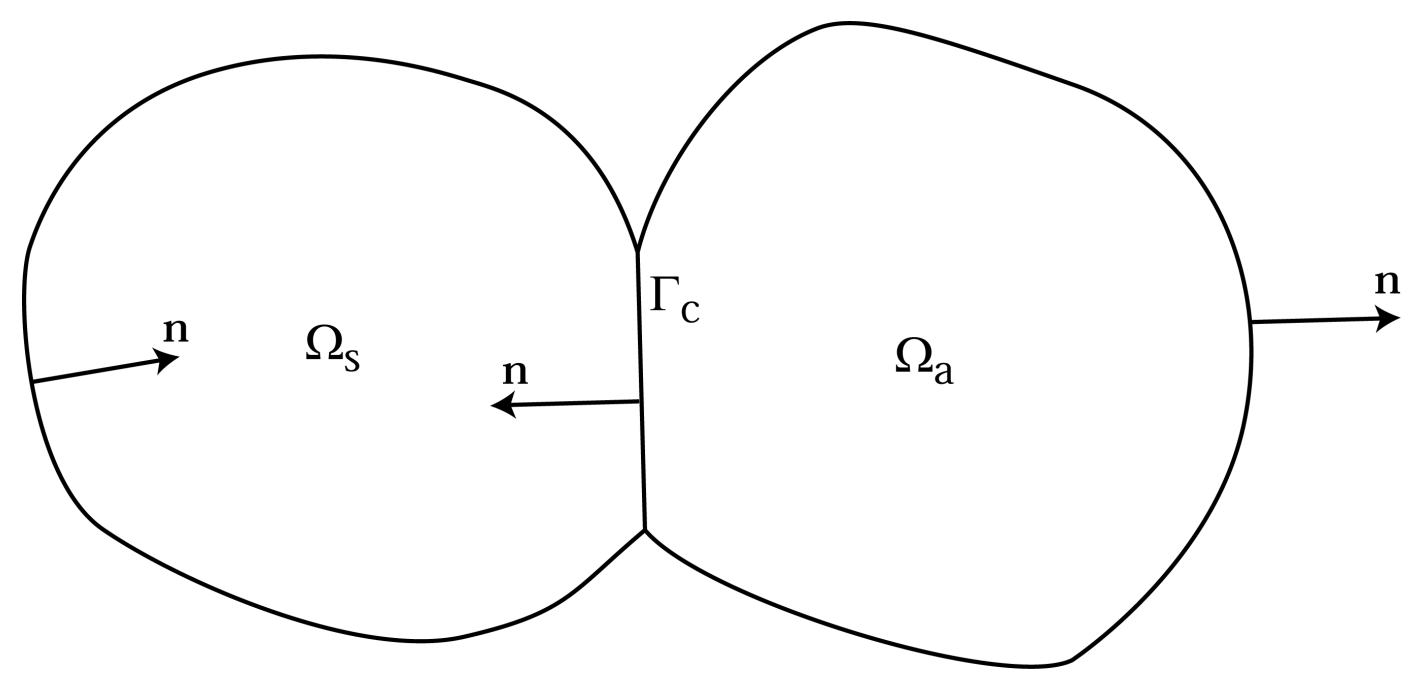

Figure 1. Sketch of the fluid-structure interaction problem. 

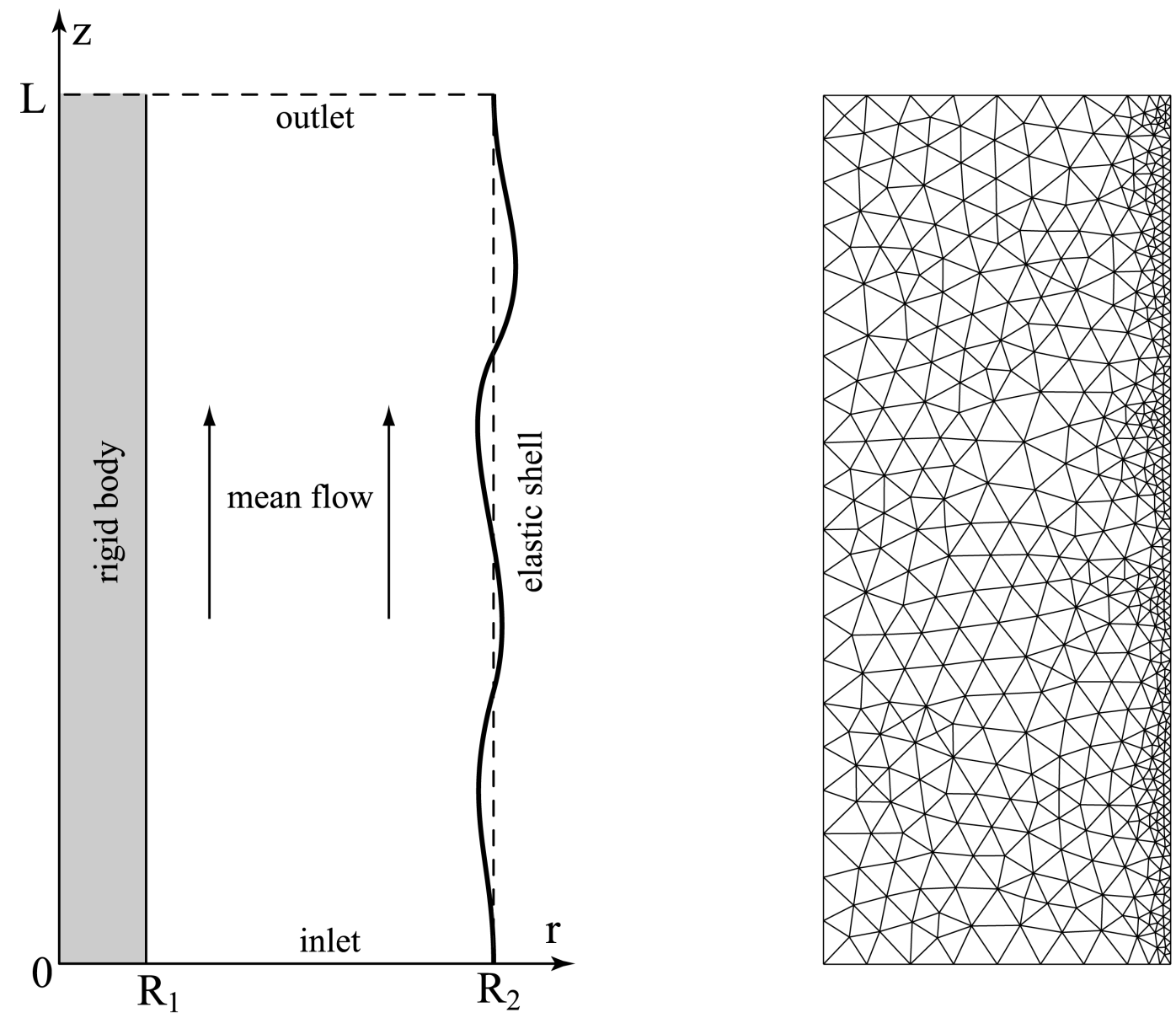

Figure 2. Sketch of the validation problem (left); Finite element mesh (right). 

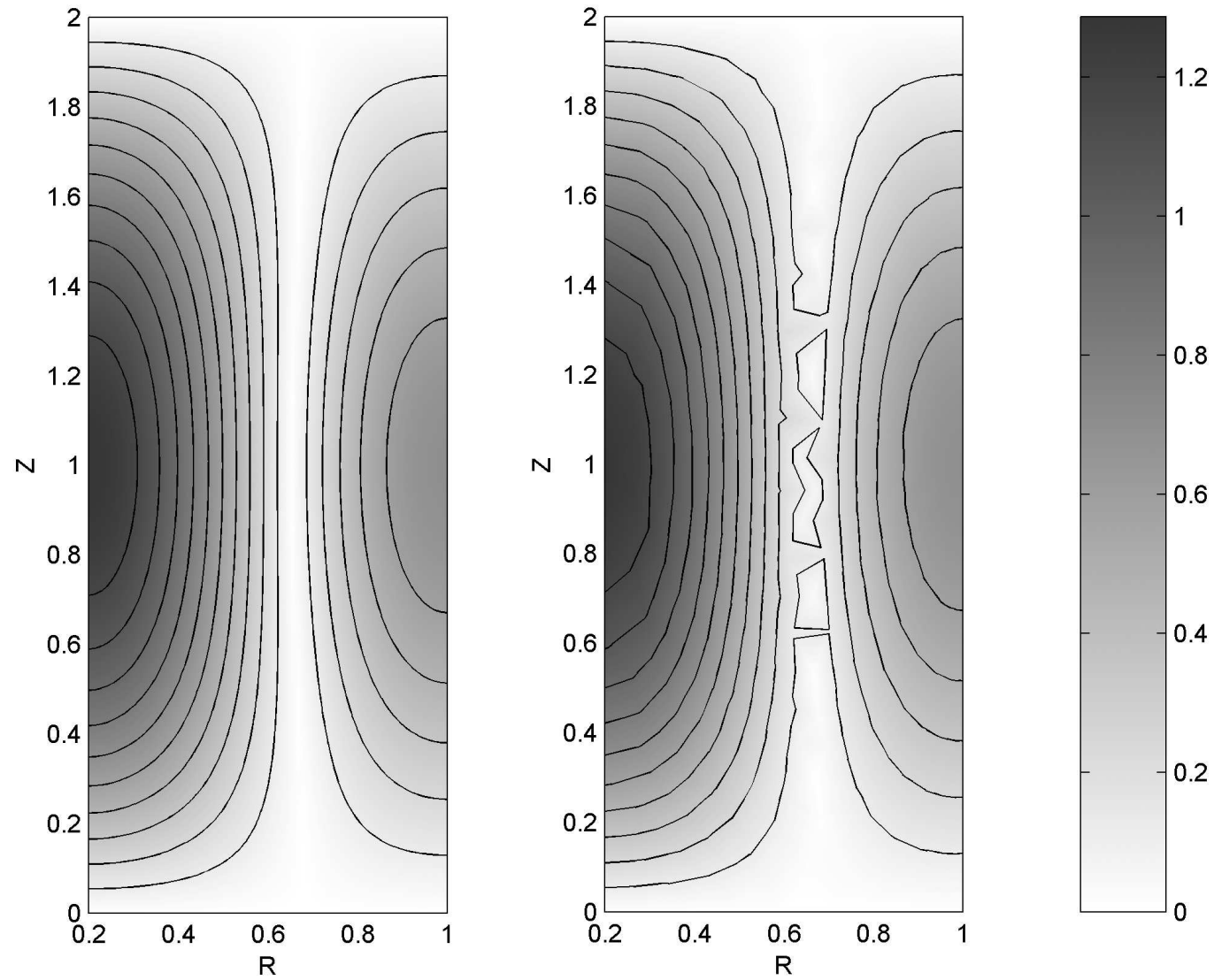

Figure 3. Acoustic pressure amplitude (in $\mathrm{Pa}$ ) for the first validation problem with no flow at 245Hz: Analytical model (left); Numerical model (right). 

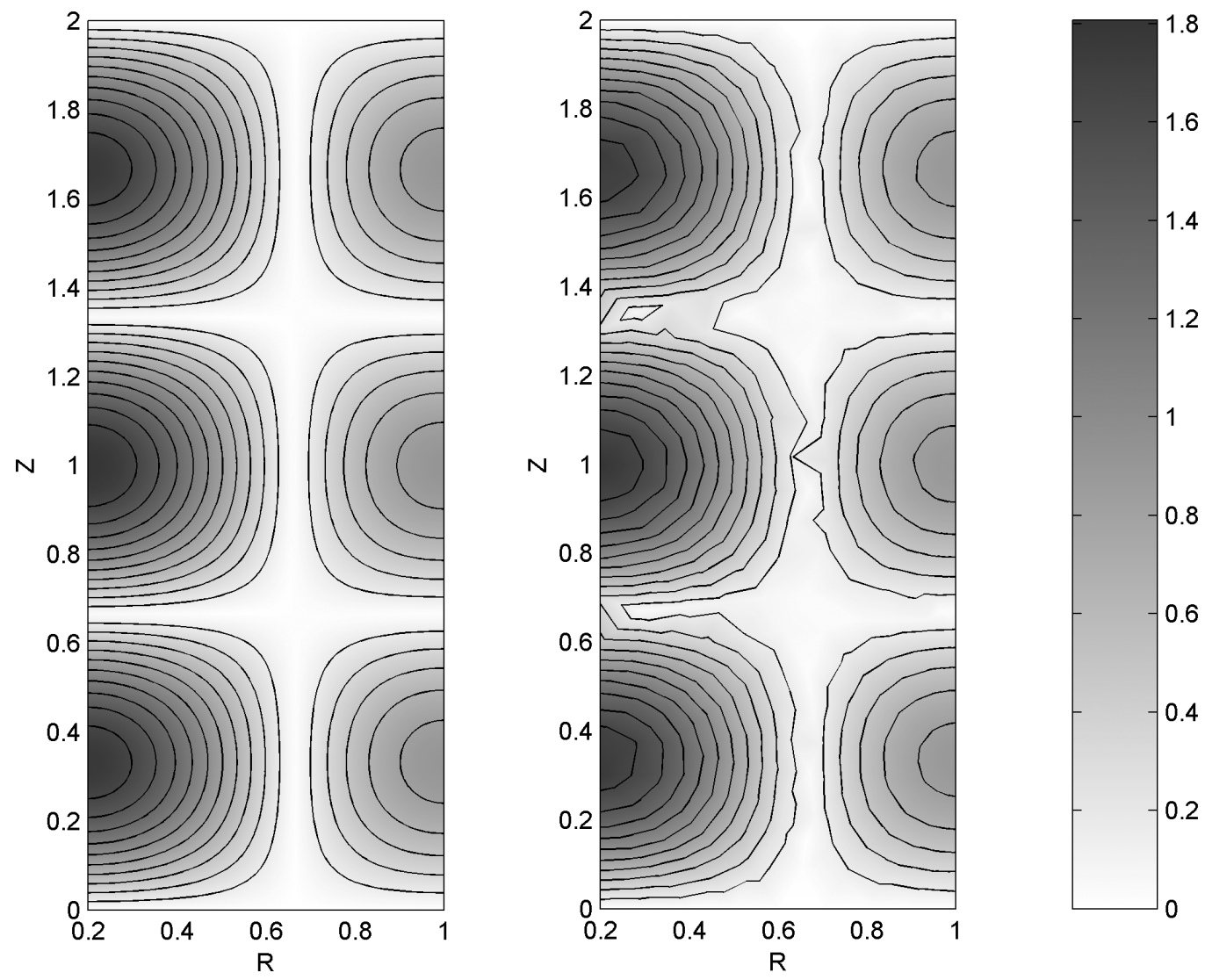

Figure 4. Acoustic pressure amplitude (in $\mathrm{Pa}$ ) for the first validation problem with a $0.3 \mathrm{Mach}$ number uniform flow at 318Hz: Analytical model (left); Numerical model (right). 


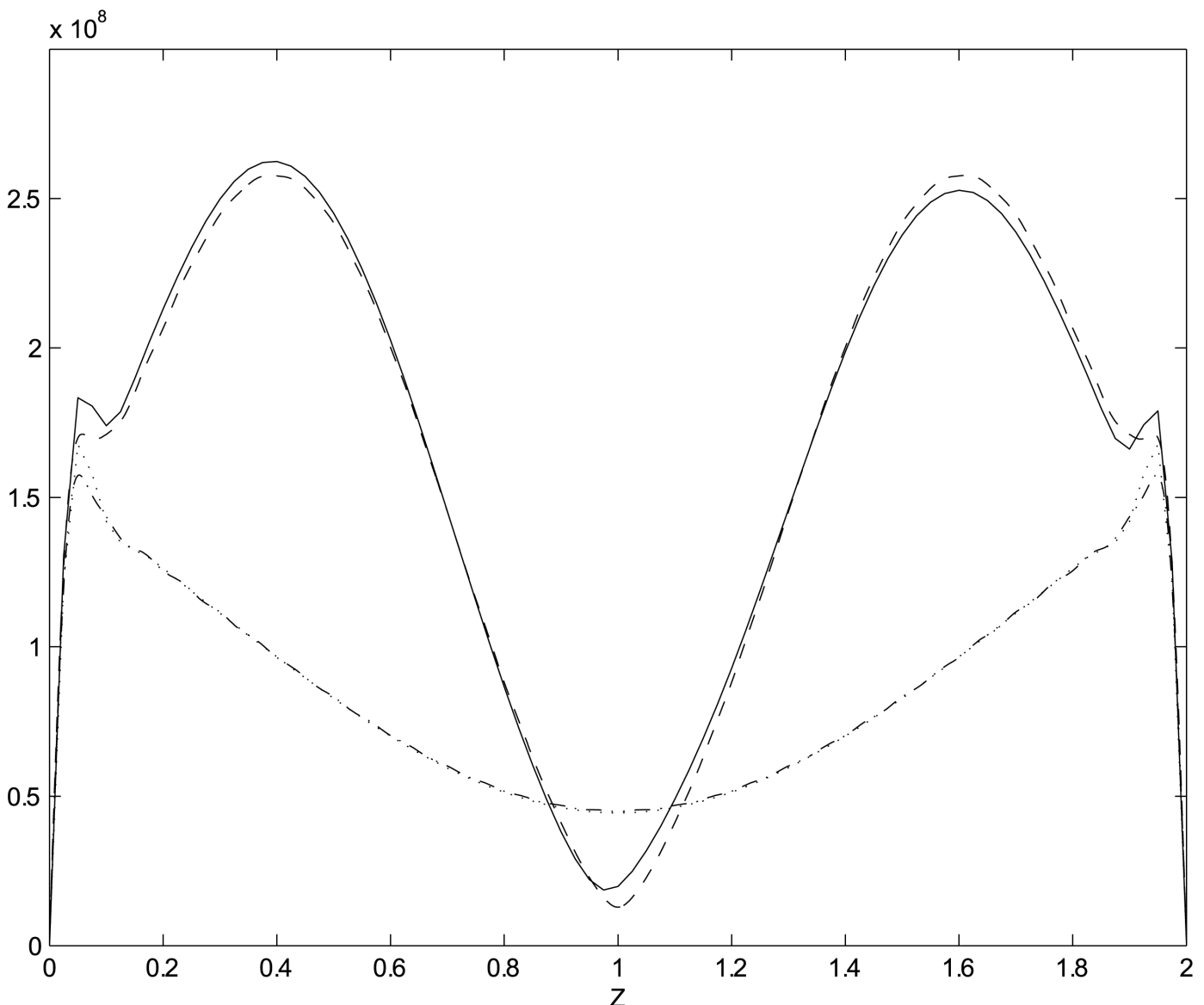

Figure 5. Radial structural displacement amplitudes (in $\mathrm{m}$ ) for the first validation problems. With no flow at 245Hz: analytical model (dot-dash line), numerical model (dotted line). With a 0.3 Mach number uniform flow at 318Hz: analytical model (dashed line), numerical model (solid line). 

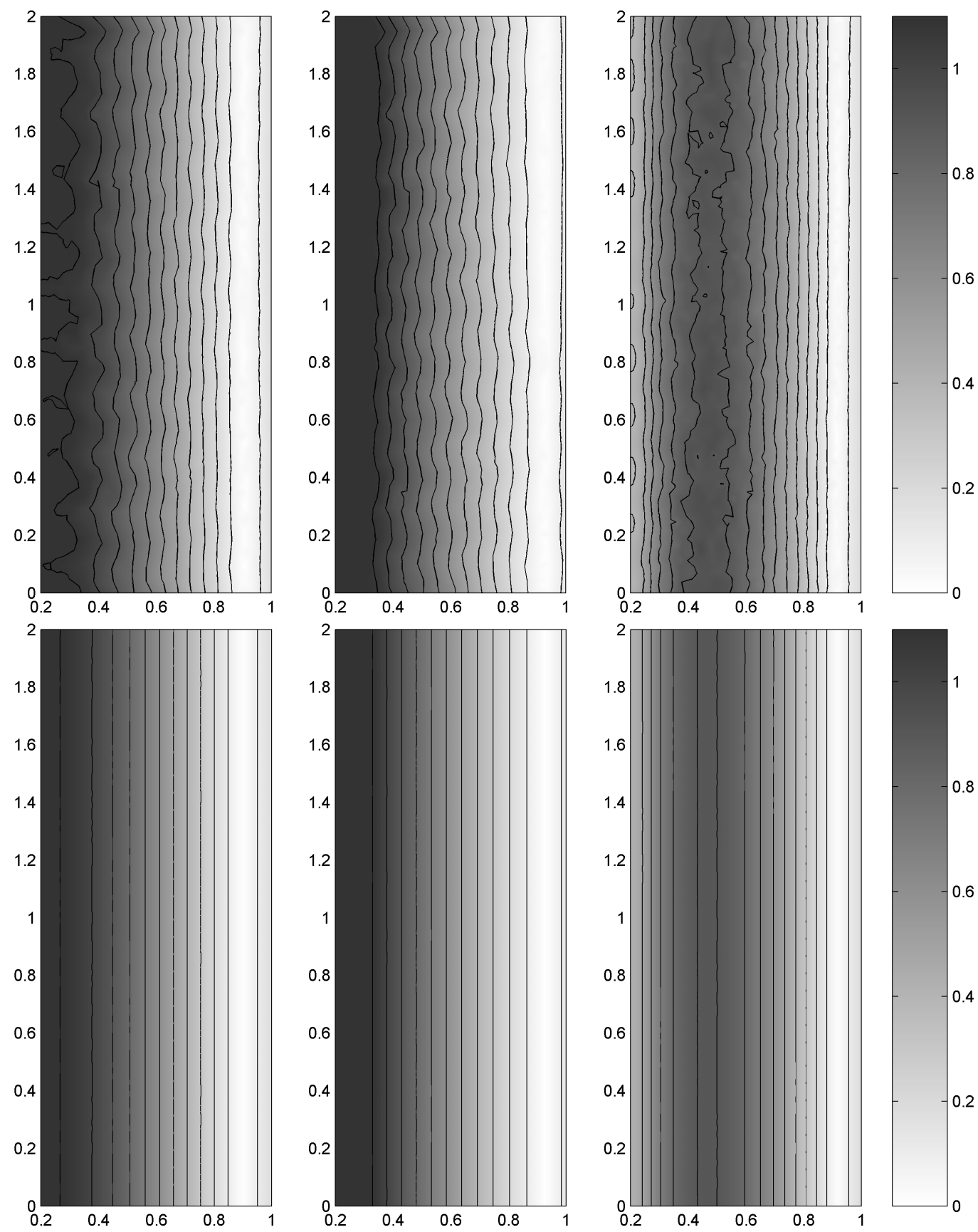

Figure 6. Acoustic pressure amplitudes (in $\mathrm{Pa}$ ) for the second validation problem. Results obtained with the finite element model (top) and the analytical model (bottom): downstream propagation with an uniform mean flow (left), downstream propagation with a sheared mean flow (center) and upstream propagation with a sheared mean flow (right). 


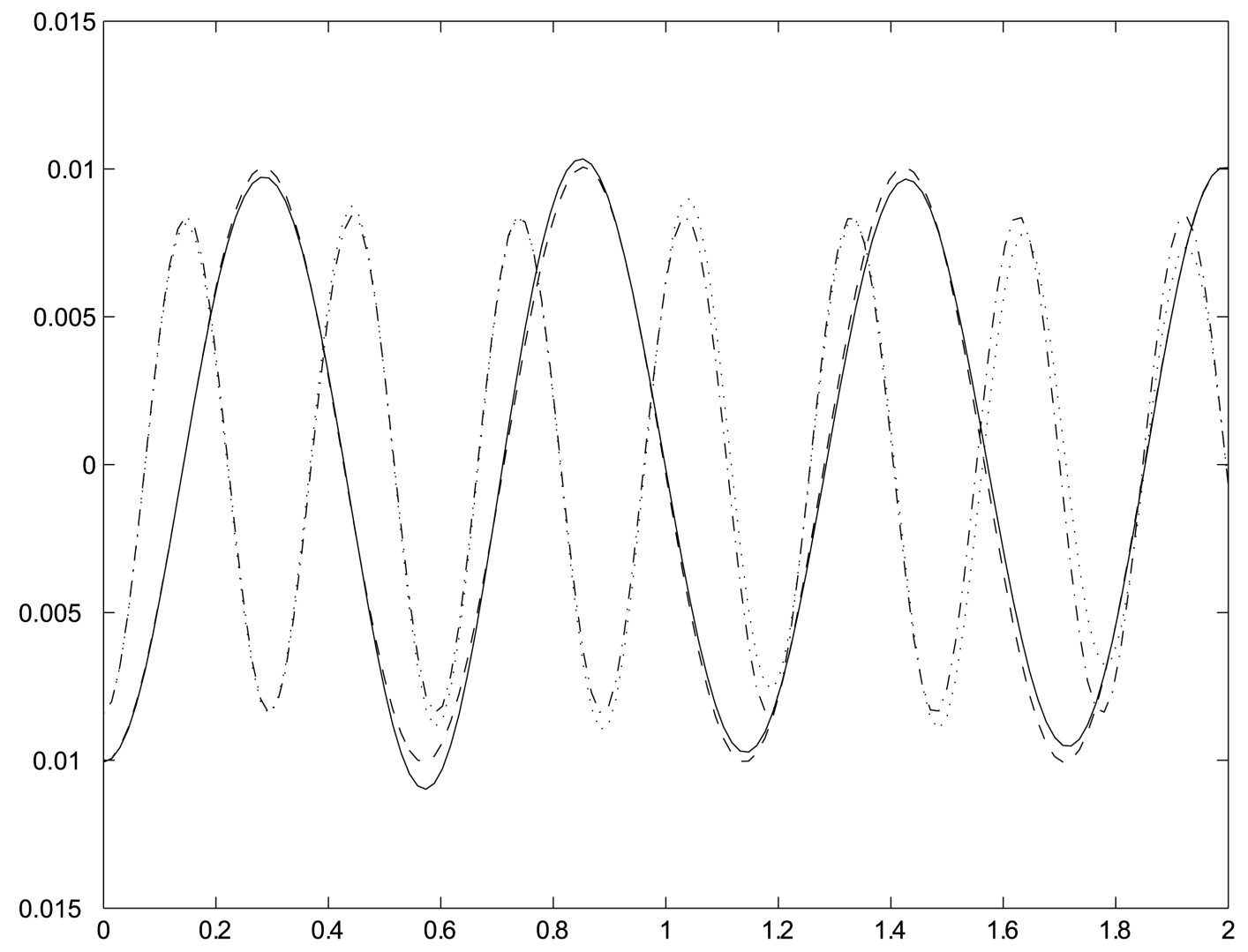

Figure 7. Radial structural displacement amplitudes (in $\mathrm{m}$ ) for the second validation problems. Downstream propagation in a sheared mean flow: analytical model (dashed line), numerical model (solid line). Upstream propagation in a sheared mean flow: analytical model (dot-dash line), numerical model (dotted line). 

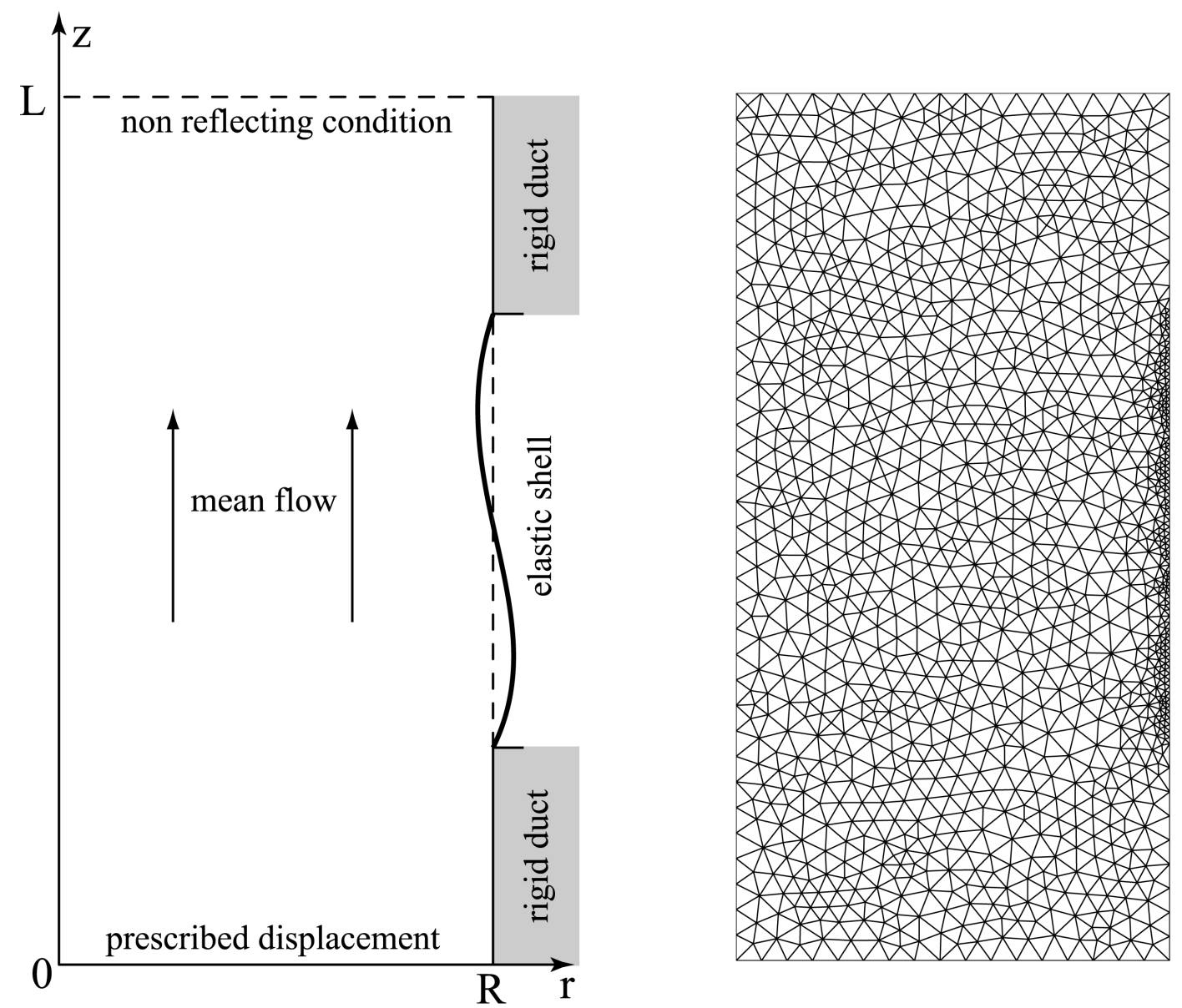

Figure 8. Sketch of the example (left); The finite element mesh (right). 

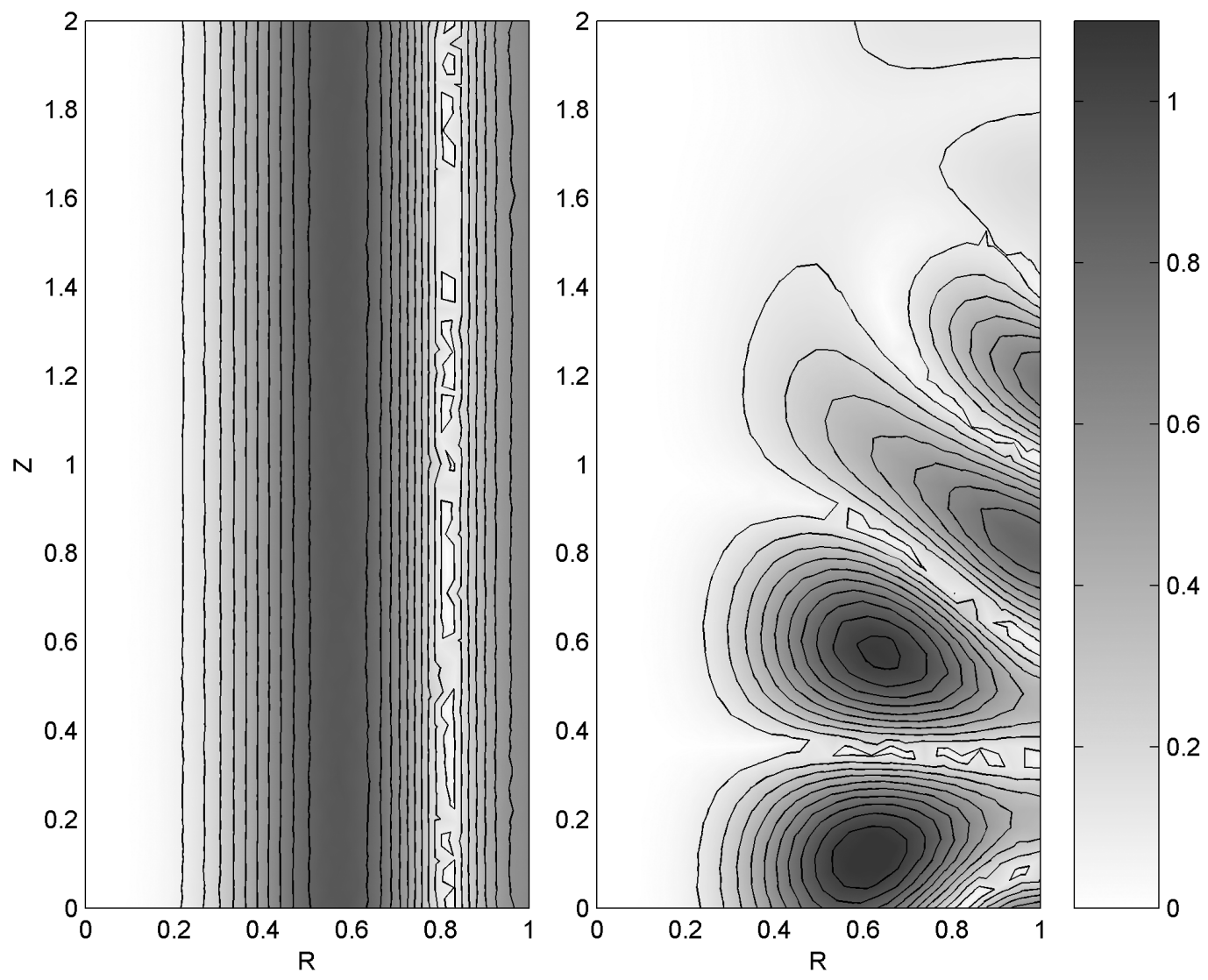

Figure 9. Acoustic pressure amplitude (in Pa) for an incoming acoustic mode $(4,1)$ at $570 \mathrm{~Hz}$ with no flow: Rigid duct (left); Elastic duct (right). 

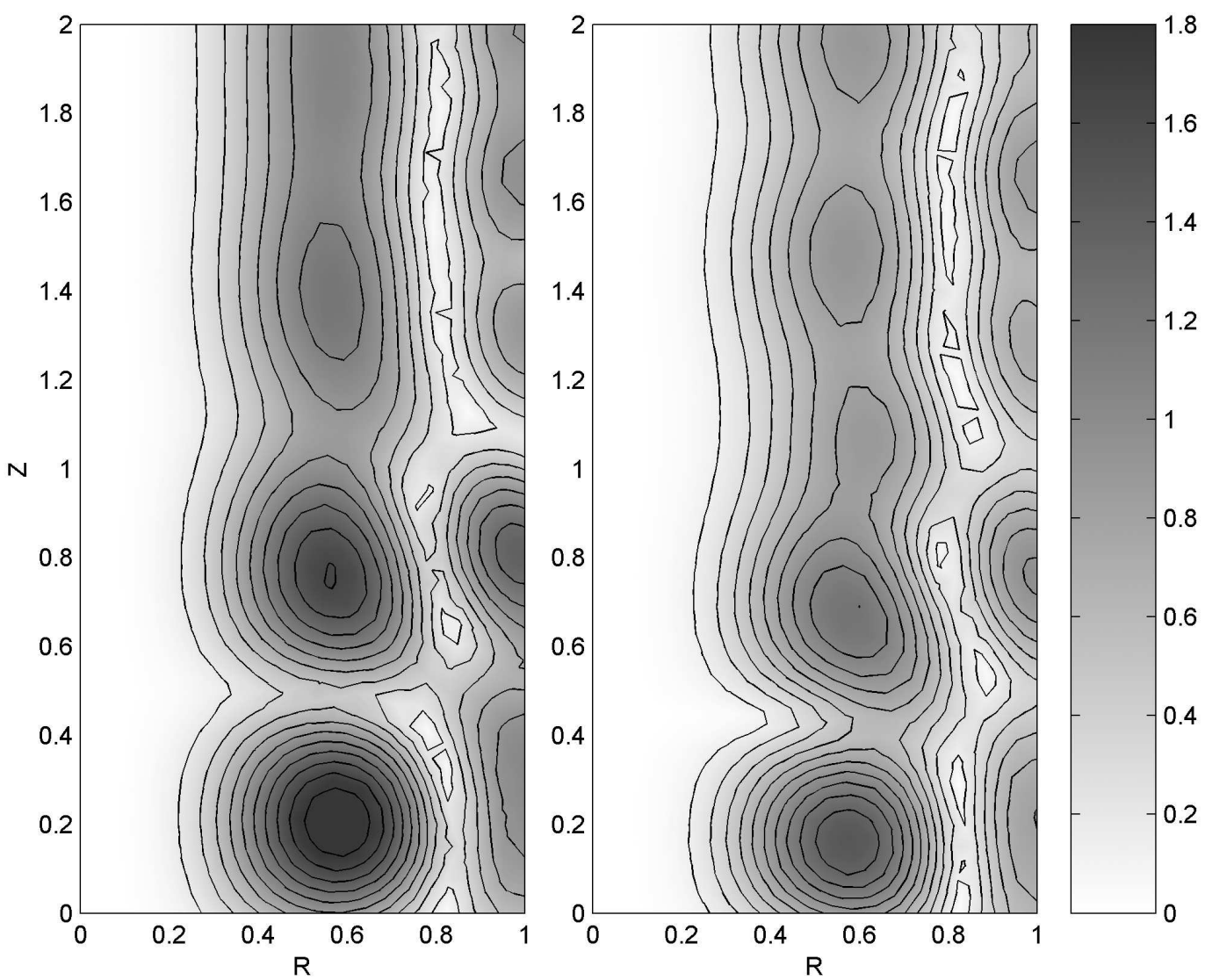

Figure 10. Acoustic pressure amplitude (in $\mathrm{Pa}$ ) for an incoming acoustic mode $(4,1)$ at $570 \mathrm{~Hz}$ with a 0.2 Mach number flow: Uniform flow (left); Parabolic flow (right). 

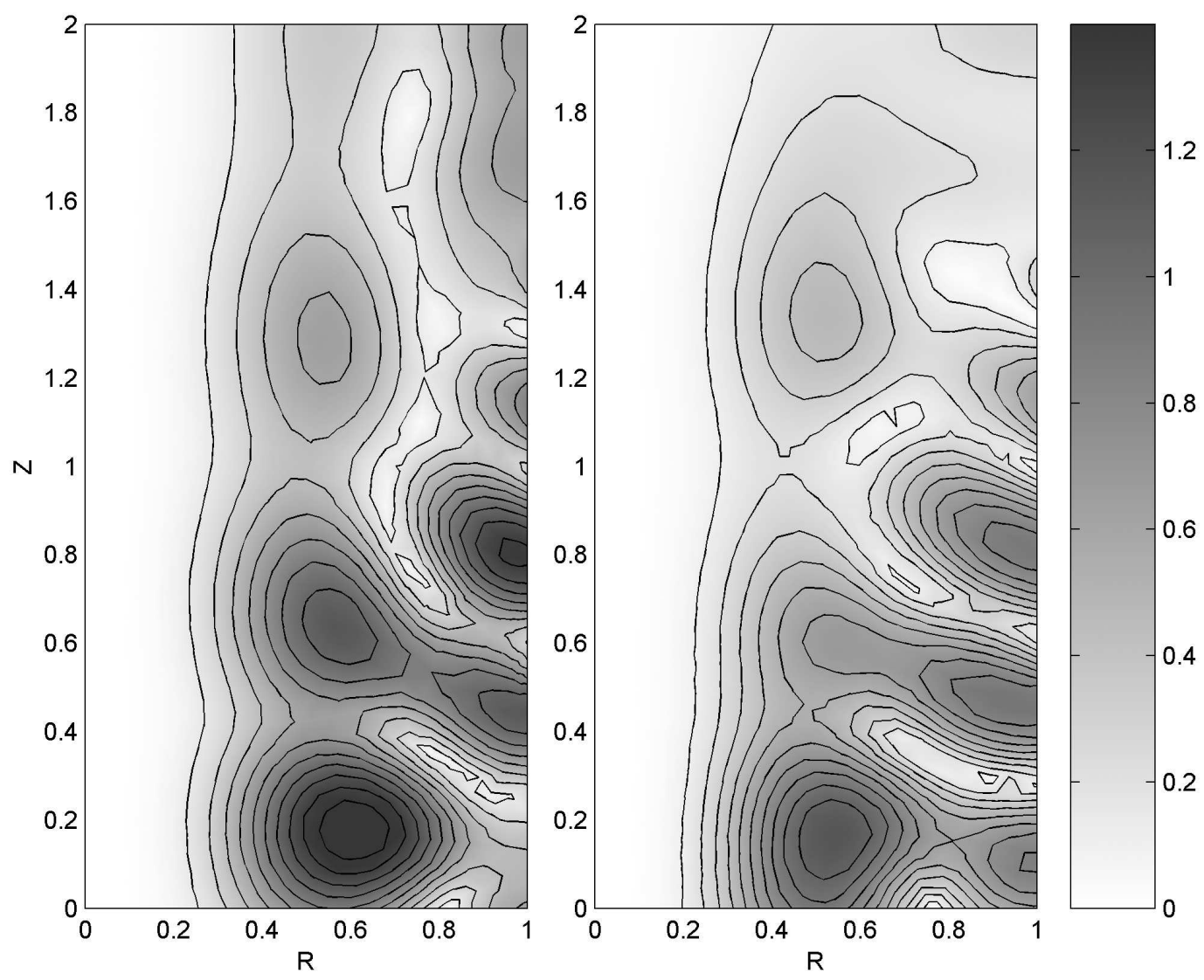

Figure 11. Acoustic pressure amplitude (in $\mathrm{Pa}$ ) for an incoming acoustic mode $(4,1)$ at $570 \mathrm{~Hz}$ with a -0.2 Mach number flow: Uniform flow (left); Parabolic flow (right). 

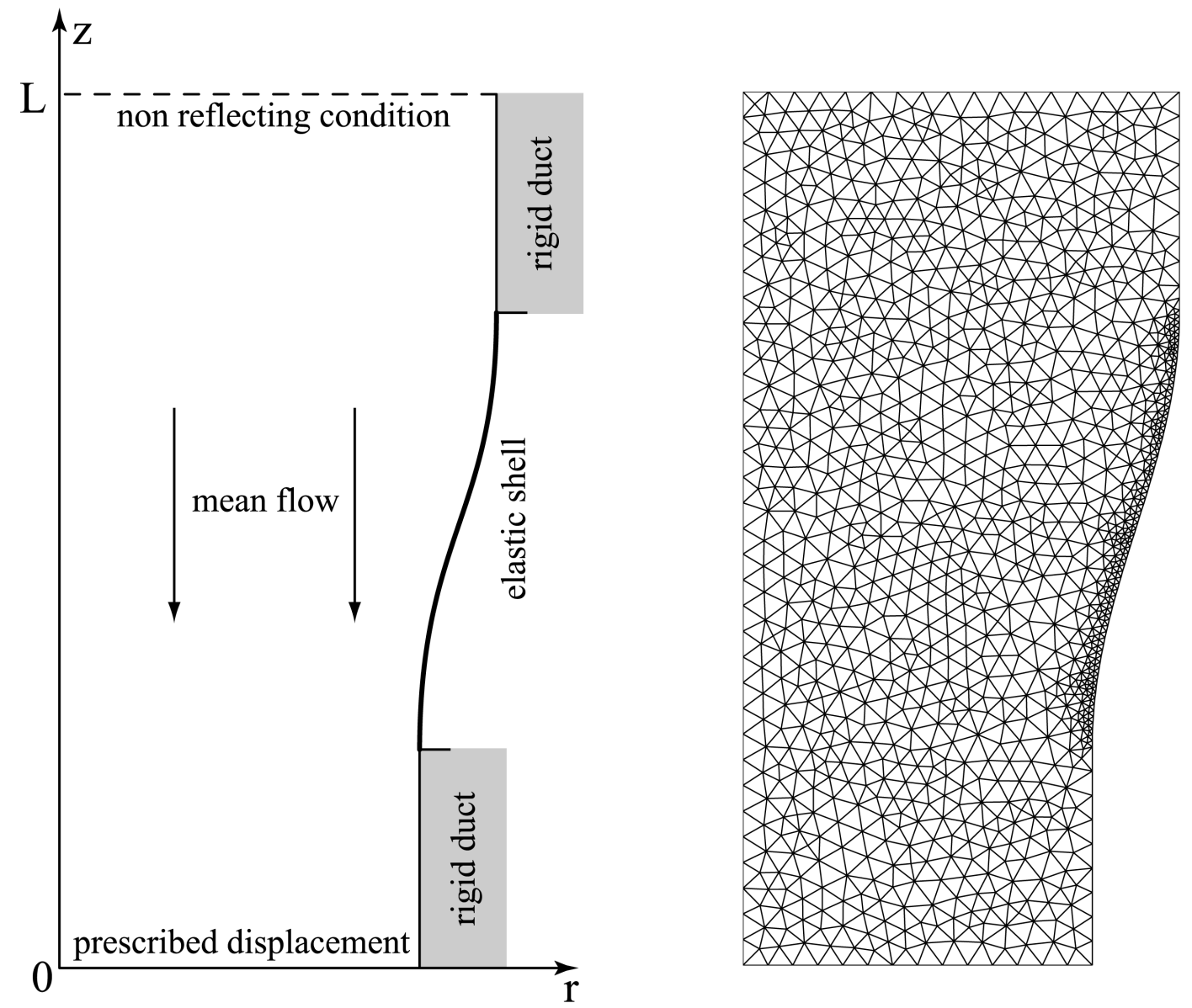

Figure 12. Sketch of the varying elastic duct (left); The finite element mesh (right). 

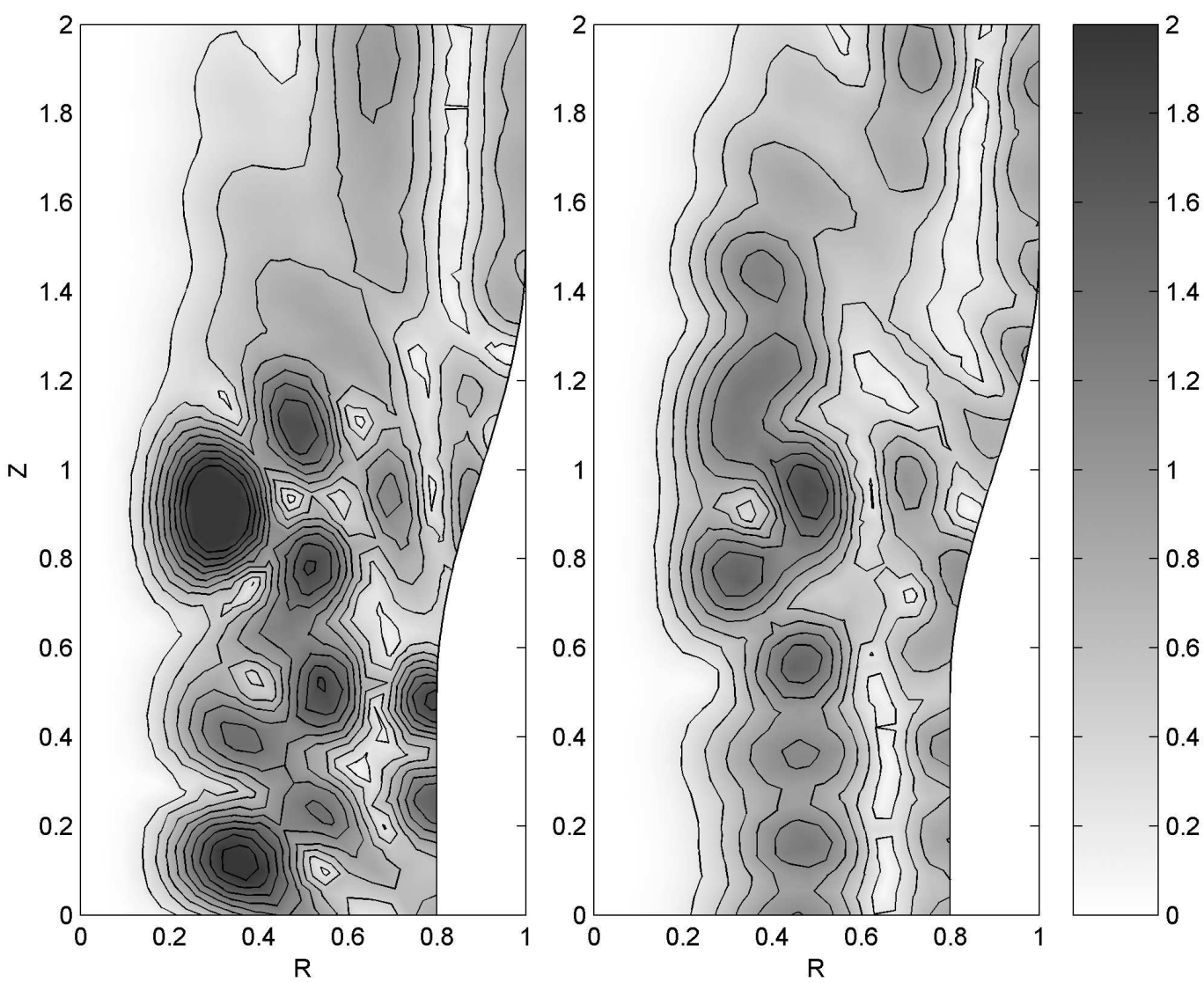

Figure 13. Acoustic pressure amplitude (in $\mathrm{Pa}$ ) for an incoming acoustic mode $(4,1)$ at $1015 \mathrm{~Hz}$ in a variable duct: With no flow (left); With a 0.2 Mach number flow (right). 
FIGURE CAPTIONS

Figure 1. Sketch of the fluid-structure interaction problem.

Figure 2. Sketch of the validation problem (left); Finite element mesh (right).

Figure 3. Acoustic pressure amplitude (in $\mathrm{Pa}$ ) for the first validation problem with no flow at 245Hz: Analytical model (left); Numerical model (right).

Figure 4. Acoustic pressure amplitude (in $\mathrm{Pa}$ ) for the first validation problem with a 0.3 Mach number uniform flow at 318Hz: Analytical model (left); Numerical model (right).

Figure 5. Radial structural displacement amplitudes (in $\mathrm{m}$ ) for the first validation problems. With no flow at $245 \mathrm{~Hz}$ : analytical model (dot-dash line), numerical model (dotted line). With a 0.3 Mach number uniform flow at $318 \mathrm{~Hz}$ : analytical model (dashed line), numerical model (solid line).

Figure 6. Acoustic pressure amplitudes (in $\mathrm{Pa}$ ) for the second validation problem. Results obtained with the finite element model (top) and the analytical model (bottom): downstream propagation with an uniform mean flow (left), downstream propagation with a sheared mean flow (center) and upstream propagation with a sheared mean flow (right).

Figure 7. Radial structural displacement amplitudes (in $\mathrm{m}$ ) for the second validation problems. Downstream propagation in a sheared mean flow: analytical model (dashed line), numerical model (solid line). Upstream propagation in a 
sheared mean flow: analytical model (dot-dash line), numerical model (dotted line).

Figure 8. Sketch of the example (left); The finite element mesh (right).

Figure 9. Acoustic pressure amplitude (in $\mathrm{Pa}$ ) for an incoming acoustic mode $(4,1)$ at $570 \mathrm{~Hz}$ with no flow: Rigid duct (left); Elastic duct (right).

Figure 10. Acoustic pressure amplitude (in $\mathrm{Pa}$ ) for an incoming acoustic mode $(4,1)$ at $570 \mathrm{~Hz}$ with a 0.2 Mach number flow: Uniform flow (left); Parabolic flow (right).

Figure 11. Acoustic pressure amplitude (in $\mathrm{Pa}$ ) for an incoming acoustic mode $(4,1)$ at $570 \mathrm{~Hz}$ with a -0.2 Mach number flow: Uniform flow (left); Parabolic flow (right).

Figure 12. Sketch of the varying elastic duct (left); The finite element mesh (right).

Figure 13. Acoustic pressure amplitude (in $\mathrm{Pa}$ ) for an incoming acoustic mode $(4,1)$ at $1015 \mathrm{~Hz}$ in a variable duct: With no flow (left); With a 0.2 Mach number flow (right). 